\title{
Economic Growth Under Shocks: Path Dependencies and Stabilization
}

Yuri M. Ermoliev, Tatiana Y. Ermolieva, and Vladimir I. Norkin

RP-06-001

March 2006

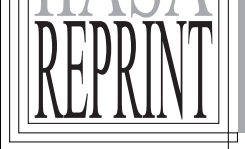





\title{
Economic Growth Under Shocks: Path Dependencies and Stabilization
}

\author{
Yuri M. Ermoliev \\ International Institute for Applied Systems Analysis, Laxenburg, Austria \\ Tatiana Y. Ermolieva \\ International Institute for Applied Systems Analysis, Laxenburg, Austria \\ Vladimir I. Norkin \\ Glushkov Institute of Cybernetics, Kiev, Ukraine
}

RP-06-001

March 2006

Reprinted from MICRO MESO MACRO: Addressing Complex Systems Couplings, Hans Liljenström and Uno Svedin, Editors, World Scientific Publishing Co. Pte. Ltd., Singapore. 
IIASA Reprints make research conducted at the International Institute for Applied Systems Analysis more accessible to a wider audience. They reprint independently reviewed articles that have been previously published in journals. Views or opinions expressed herein do not necessarily represent those of the Institute, its National Member Organizations, or other organizations supporting the work.

Reprinted from MICRO MESO MACRO: Addressing Complex Systems Couplings,

Hans Liljenström and Uno Svedin, Editors.

Copyright $@$ 2005, with permission from World Scientific Publishing Co. Pte. Ltd., Singapore.

All rights reserved. No part of this publication may be reproduced or transmitted in any form or by any means, electronic or mechanical, including photocopy, recording, or any information storage or retrieval system, without permission in writing from the copyright holder. 


\title{
Chapter 15
}

\section{Economic Growth Under Shocks: Path Dependencies and Stabilization}

\author{
Yuri M. Ermoliev, Tatiana Y. Ermolieva \\ \& Vladimir I. Norkin
}

\section{Introduction}

Increasing vulnerability of the modern society is an alarming tendency of current global changes. Losses from human-made and natural catastrophes are rapidly increasing (Climate Change, 1999). For example, within the last two decades the direct catastrophe damages from only natural disasters have increased nine-fold. Catastrophes destroy communication systems, the electricity supply and the irrigation. They affect consumption, savings and investments. The main reason for the increasing catastrophe losses is the lack of appropriate information on risks leading to the clustering of values in risk prone areas and the creation of new risk prone areas. The standard economic theory is based, in fact, on rather strong assumptions of certainty. It is assumed that economic agents know all possible shocks (states of the world), i.e., they know when, how often, and what may happen to each of them. Therefore, they can easily pool resources available in any state of the entire society, i.e., a catastrophe becomes small on the scale of the world (Arrow, 1996). In reality this pool does not exist, which calls for more realistic models with explicit representation of uncertainties and associated risks. Especially important are models that explain connections between poverty, stagnation, and shocks. One of the reasons which could cause low growth (Easterly, 1994), (Solow, 1997) is the low saving rates typically observed in low-income countries: economies where the majority of citizens have incomes close to minimum of subsistence level are unlikely to have a high rate of savings. Obviously, shocks affect savings, but the low saving rate is not sufficient to explain within conventional deterministic models why the sustained growth may not "take-off".

This paper analyzes effects of random shocks on economic growth and stagnation. A shock is understood as an event destroying a part of capital 
stock. For example, shocks may be due to the flight of capital from the country. We show that on average the economy may still grow exponentially, but actual growth path exhibits stagnation, shrinking and even traps. Fundamental deterministic and stochastic models of economic growth are nicely discussed in (Sargent, 1987), (Solow, 1997). In our model we analyze effects of endogenously generated shocks when their impacts (probability distribution) depend on the level of economic developments. Shocks of this type are responsible for the increasing catastrophic losses and the vulnerability of interacting socio-economic and natural systems. Section 4 illustrates that even in the case of the exponential growth, persistent in time shocks may implicitly modify the production function of the economy and lead to traps and path dependencies.

The stabilization of growth has to rely on deliberately designed anticipative (forward looking, ex-ante) and adaptive (backward looking, ex-post) defensive strategies such as borrowing, loss reduction measures, insurance and financial markets. As the economy grows crossing certain thresholds (instability levels), the assistance for growth can be reduced or completely disappear. Section 2 analyzes effects of shocks on sustained growth. In section 3 we illustrate that ex-ante and ex-post policies for dealing with shocks are not substitutes and must be analyzed jointly as complementary decisions. The outlined in this section and section 5 a two-stage dynamic stochastic programming model incorporates both ex-ante and ex-post decisions. It essentially differs from models of the standard control theory through the presence of anticipative decisions, and it differs from the traditional models of the expected utility theory through the presence of adaptive decisions. Qualitatively, this model provides more realistic decision making framework according to which only some decisions are made ex-ante, whereas other options are kept open until more information about shocks becomes available and, hence, can be better utilized in ex-post decisions. In discussed models a strong risk aversion occurs even for linear utility functions. Section 4 illustrates the convergence of an economy under shocks to a path of sustained growth. Shocks implicitly modify the economy, and the convergence becomes path dependent. The economy may stagnate without appropriate growth efforts to by-pass various traps and thresholds. Section 5 contains more detailed mathematical formulation of the outlined in sections 3 and 4 models. Section 6 concludes. 


\section{Exponential economic growth}

Let us use some mathematical notations to simplify the otherwise very lengthy explanations. The ability to grow in our simple conceptual model is characterized by the production function with two factors: "capital" and "labor", $Y=F(K, L)$, and constant returns to scale, $Y=L F(K / L, 1)$, where $Y$ is its output. Therefore, we can characterize the economy in terms of capital to labor ratio, $k=K / L$, and output to labor ratio, $y=Y / L, y=f(k):=F(k, 1)$. Assume that output $Y$ is subdivided into consumption and savings, and savings are equal to investments $I$. The growth is driven by the accumulation of capital through investments

$$
\frac{d K}{d t}=I-\delta K, K(0)=K_{0}, t>0
$$

where $\delta, 0<\delta<1$, is the capital depreciation rate. Assume further that the investments $I(t)$ are simply a fraction $s, 0<s<1$, of the output, i.e., $I(t)=s Y$ $(t)$, and $\gamma$ is an exponential growth rate of the population, $d / d t \ln L=\gamma$. We can then rewrite (1) in variables $k$ :

$$
\frac{d k}{d t}=s f(k)-(\gamma+\delta) k, k(0)=k_{0}, t>0
$$

or

$$
\frac{d}{d t} \ln k=s \frac{f(k)}{k}-\gamma-\delta
$$

If the output to capital ratio is constant $\theta$, i.e., $y / k=f(k) / k=\theta$, then it leads us to the very influential Harrod-Domar (Ray, 1998), (Solow, 1997) model with constant exponential rate of growth

$$
\frac{d}{d t} \ln k=s \theta-\gamma-\delta
$$

According to (4), the rate of growth is determined jointly by the saving rate $s$ and the productivity of capital $\theta$, that is, the inverse of the capital-output ratio. From $y / k=\theta$ follows that the growth in real output $d / d t \ln y(t)$ is the same as the growth in the capital stock $d / d t \ln k(t)$. Therefore, the exponential growth is defined by linear function

$$
\ln y=\ln y_{0}+(s \theta-\gamma-\delta) t, y(0)=y(0)=y_{0}, t>0 \text {. }
$$


The economy is a complex system constantly facing shocks and changes. A catastrophe is one of such shocks. We can model shocks similarly to the depletion of capital reducing the rate of growth $s \theta-(n+\delta)$ by a random level $v=v(t, y, \omega)$, where $v(t, y, \omega)$ denotes impacts of the shock $\omega$ at the current $y(t)$. Shocks occur at random time moments $T_{0}, T_{1}, T_{2}, \ldots, T_{0}=0, v(0, y, \omega)=0$. The random intensity $v$ in our model depends on the aggregate current level $y(t)$. In realistic versions of the model $v(t, y, \omega)$ depends on the geographical distribution of the wealth and shocks as well as on other country-specific sources of vulnerability. The accumulation of investments in specific risk prone regions and sectors of the economy can make significant difference to the probability distribution of $v$. The instability of the economy due to shocks, say, from natural disasters, may provoke the flight of capital from the country and further magnify the instability. Shocks, in general, transform linear function (5) into highly nonlinear (discontinuous) random function (see Fig. 1)

$$
\ln y(t)=\ln y_{0}+(s \theta-\gamma-\delta) t-V(t), V(t)=\sum_{t=1}^{N(t)} v_{i},
$$

where $N(t)$ is the number of shocks in the interval $(0, t]$, and $v_{i}$ is the size of these shocks.

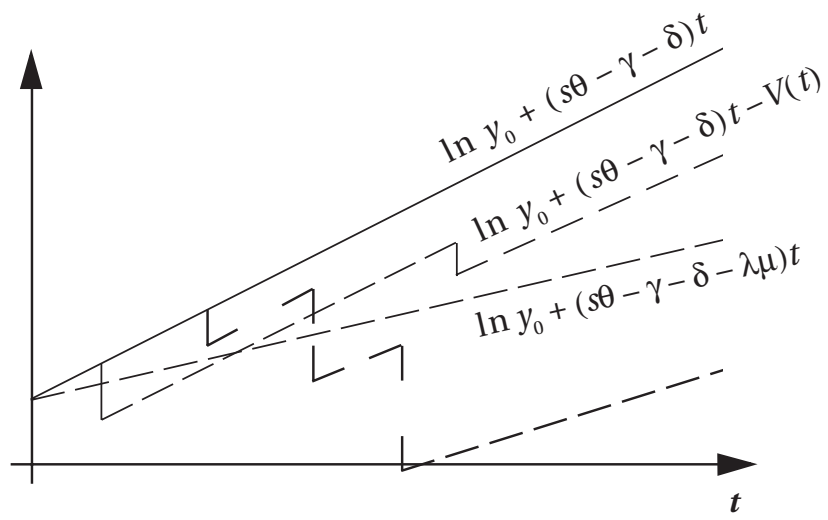

Figure 1. Expected and real growth rates.

Assume that random variables $v_{1}, v_{2}, \ldots$ are independent, identically distributed, and they are independent of intershock times $\tau_{i}=\mathrm{T}_{i}-\mathrm{T}_{i-1}$. If intershock times have stationary distribution with mathematical expectation $\lambda$, then $E \ln y(t)=\ln y_{0}+(s \theta-\gamma-\delta-\lambda \mu) t$. Since, $\ln E y(t)>E \ln y(t)$, then the expected exponential growth is still characterized by a linear in 
$t$ function (see Fig. 1) and it is positive when the rate of investments exceeds the average rate of losses, $s \theta-\gamma-\delta-\lambda \mu>0$. At the same time real random growth path $y(t, \omega)$ may considerably fluctuate. From the strong law of large numbers it follows that

$$
\frac{\ln y(t, \omega)}{t} \rightarrow s \theta-\gamma-\delta-\lambda \mu
$$

with probability 1 . It means that for each possible random growth path $y(t, \omega)$ the sustained growth "takes-off" only after a random time $T(\omega)$, i.e., $\ln y(t, \omega) \approx(s \theta-\gamma-\delta-\lambda \mu) t>0, \forall t>\mathrm{T}(\omega)$. On the way to the sustained growth for $t<T(\omega)$ the economy may stagnate and even shrink, i.e., it requires assistance.

Thus, for a given $t$ there may exist a positive probability that accumulated random losses exceed accumulated growth

$$
(s \theta-\gamma-\delta) t-V(t) \leq 0 .
$$

This probability characterizes the vulnerability of the growth, whereas the probability of the complementary event characterizes its resilience. The ability of the growth to sustain a given target rate $g$ can be characterized by the probability that the accumulation of growth between successive (say, $t$ and $t+\tau)$ shocks exceeds possible losses, i.e.,

$$
(s \theta-\delta-\gamma) \tau-V(t, \tau) \geq q \tau,
$$

where $V(t, \tau)$ are losses associated with the first shock which occurs at time $t+\tau$ after $t$. Let us note that the deterministic model characterized by the linear function $\ln y_{0}+(s \theta-\gamma-\delta-\lambda \mu) t$ is not able to address these issues since it ignores the variability of the real growth path, i.e., it ignores the risk of growth. In our simple model this is equivalent to the substitution of the complex jumping process $\ln y(t)$ by the linear function

$$
\ln y_{0}+(s \theta-\gamma-\delta-\lambda \mu) t .
$$

This function still shows the exponential growth with increased depreciation rate $\delta+\lambda \mu$, but it ignores possible stagnation of the economy in the interval $[0, T]$. The uncertainty analysis, which is usually recommended after such deterministic substitutions, can not reveal the possibility of the stagnation either, since in our case it is equivalent to the turning around the function (9). 


\section{Stabilization of growth: Anticipative and adaptive strategies}

The common practice to deal with economic shocks is to use adaptive (expost) measures as borrowing, foreign aid, and the diversion of investments committed to other needs. This section illustrates the importance of both anticipative (ex-ante) and adaptive (ex-post) control strategies in order to be prepared for shocks before they occur, as well as to have enough flexibility to react more effectively to revealed situations.

The deterministic Harrod-Domar model (4) is a building block in the growth control (Ray, 1998), (Khan et al., 1990). According to (4), the overall rate of growth in the interval $[t, t+\Delta t]$ for small enough $\Delta t$ is approximately defined as

$$
[Y(t+\Delta t)-Y(t)] / \Delta t Y(t) \approx s \theta-\gamma-\delta .
$$

This equation links the growth rate of the economy to two fundamental variables: the ability of the economy to save and the productivity of the capital $\theta$. By increasing the rate of savings $s$ or capital productivity $\theta$, it would be possible to accelerate the rate of growth. This creates the basis for programming of the growth rate, i.e., the design of policies which provide such levels of parameters $s, \theta, \gamma, \delta$, that guarantee a given level of growth g. A "gap" between available investments and the investment required to achieve the output growth target, e.g., due to "unforeseen" shock, provides the information for the borrowing needed to cover this "gap".

Equation (10) has rather conceptual, symbolic character. In realistic models values of all parameters $s, \theta, \gamma, \delta$ are defined by various components. For example, the distribution of incomes among the population and investments among various sectors of the economy and geographical regions may play a critical role for the stability of the growth. If some of the parameters $s, \theta, \gamma, \delta$ or their components are fixed, then missing values can be defined from (10). As it was pointed out in (Ray, 1998, p. 58), such "if-then" analysis in many cases does not make sense, since the parameters that are used to predict growth rate may themselves be affected by the growth processes. Besides this, a critical issue arises with the naïve adaptive sequential adjustments of growth rates. It is evident that the analysis of the growth rate at time $t$ only for the next interval $[t, t+\Delta t]$ may not provide a good idea to develop preparedness and loss reduction measures for rare events. The occurrence of a shock in a small interval of length $\Delta t$ is often evaluated by negligibly small probability $\lambda \Delta t$, but the probability $\mathrm{l}-(\mathrm{l}-\lambda \Delta t)^{T / \Delta t} \approx 1-e^{\lambda T}$ of a shock 
in the interval $[0, T]$ is dramatically increasing with $T$. Therefore, the control of growth must take a long-term perspective in order to develop measures against the "unforeseen" otherwise shocks.

The coexistence of anticipative and adaptive decisions within the same model can be viewed as a rather flexible decision making framework when we ex-ante commit ourselves only to a part of possible decisions and, at the same time, we keep other options open until more information becomes available and can be effectively utilized by appropriate ex-post decisions. In other words, these decisions are not evaluated in a sequential, "one-by-one" manner, since the most important is the synergy of them. Thus a certain level of mitigation measures increasing, say, resistance of buildings to earthquakes, may essentially increase their insurability. On the other hand, the existence of insurance may enforce the mitigation measures through appropriate reductions in prices of insurance contracts.

The need for coexistence of ex-ante and ex-post decisions becomes more evident from the following simple situation. Assume that there are only two time intervals: "now", $t=0$, and "future", $t=1$. Ex-ante decision $x$ is made at $t=0$, whereas ex-post decision $y(\omega)$ is made at $t=1$ on the basis of information about impacts $v(\omega)$ of shock $\omega$ and the decision $x$. The decision $x$ stabilizes the economy from losses $v(\omega)$ below the level $x$ at the cost $c x$, i.e., $v(\omega)$ is transformed into the function

$$
v(x, \omega)= \begin{cases}v(\omega)-x, & \text { if } x \leq v(\omega), \\ 0, & \text { otherwise. }\end{cases}
$$

The ex-post borrowing $z(\omega)$ further stabilizes the economy from losses $v(x, \omega)$ at the cost $\beta(\omega) z(\omega)$. The cost-efficient stabilization minimizes expected value of the function

$$
f(x, y(\omega))=c x+E(\beta(\omega) z(\omega)), x \geq 0,
$$

where $z(\omega)=v(\omega)-x$ for $x \leq v(\omega)$. It is clear that $z(\omega)=\max \{0, v(\omega)-x\}$, i.e., $z(\omega)$ depends on $(x, \omega)$. Thus, the cost-efficient stabilization is reduced to the minimization of the function

$$
\begin{aligned}
F(x) & =c x+E(\beta(\omega) \max \{0, v(\omega)-\mathrm{x}\}) \\
& =c x+\int_{x}^{\bar{v}} \beta(v)(v-x) \varphi(v) d v
\end{aligned}
$$


for $x \geq 0$. Here we assume that the probability distribution of $v$ has the support $[0, \bar{v}]$, with a continuous density function $\varphi(v)$. From this it follows that $F(x)$ has the continuous derivative $F^{\prime}(x)$ and, as it is easy to verify, $F^{\prime}(x)=c-$ $\int_{x}^{y} \beta(v) \varphi(v) d v$. A solution of this problem may be $x=0$, i.e., the stabilization totally relies on the borrowing. The function $F^{\prime}(x)$ is monotonically increasing for $x \rightarrow \bar{v}$. Therefore, if $c<\int_{0}^{\bar{v}} \beta(v) \varphi(v) d v$, or $c<E \beta(v)$, then there is a positive value $x=x^{*}, x^{*} \neq \bar{v}$, such that $F^{\prime}\left(x^{*}\right)=0$. Here $x^{*}=\bar{v}$ is excluded because $c>0$. If $\beta(\omega)$ does not depend on $\omega, \beta(\omega)=\beta$, then $x^{*}$ is a quantile or the so-called Value-at-Risk (VaR) of $v(\omega)$ satisfying the equation (Ermoliev \& Wets, 1988, p. 416)

$$
P[v>x]=\frac{c}{\beta} .
$$

Thus, the minimization of the linear in $(x, y(\omega))$ function $f(x, y(\omega))$ does not lead to the dominance of the preferable on average ex-ante solution $(c<\beta)$, i.e., both ex-ante and ex-post solutions coexist.

Let us note that the notion of an anticipative solution $x$ reflects the fact that this type of solutions can not be chosen after the observation of shock $\omega$. For example, the decision $x$ may be a height of a water wall that must protect a region from floods. This type of decisions restricts the use of standard recursive equations (Sargent, 1987) of the optimal control theory dealing only with the "adaptive" control strategies. The design of combinations of ex-ante and ex-post strategies requires the use of stochastic optimization methods and models discussed with more details in section 5 .

\section{Convergence, traps and path dependencies}

Section 2 shows that even for well-defined economies with expected exponential growth rate the sustained growth takes off only in the long run. This property of the growth path radically changes when parameters $s, \theta, \gamma$ and losses $v_{i}$ are affected by the growth. In our model it is characterized by per capita income $y$. The rate of savings $s$ may critically depend on the overall level $y$ and its distribution in the society. Obviously, at low level of income, rates of savings are small. In this case a shock may further reduce them even to negative values (borrowing). As the economy grows there is increasing room for savings (Ray, 1998, p. 59), but this does not necessarily mean that savings will grow steadily. To endogenize the dependence of $s, \theta, \gamma, \delta$, on $y$ and $\omega$ requires certain assumptions, the most important of them is the assumption of utility maximizing consumers. We do not follow this approach 
in the paper. Instead, let us make a rather optimistic assumption that shocks do not affect the parameters $s, \theta, \gamma, \delta$ of the economy but only the state $y$ reducing $y$ to a level $\tilde{y}, 0<\tilde{y} \leq y$. The probability distribution of $\tilde{y}$ in the inter$\operatorname{val}[0, y]$ is characterized by a density function $\Psi$, i.e., the probability of losses in the interval $[h, h+d h]$ is $\Psi(y, h) d h$. Then the expected losses $\mu$ depend on $y$ and have the form $\mu(y)=\int_{0}^{y} h \Psi(y, h) d h$. It is easy to see that the second derivative $\mu$ " $(y)$ involves the derivative $d \Psi(y, y) / d y$, which may be positive or negative at different levels of $y$. Therefore $\mu$ " $(y)$ may have an oscillating character, as it is shown in Fig. 2. From (6) follows that for the shocks independent of the intershocks times, the change of $y(t)$ to a value $y(t+\Delta t)$ in the interval $[t, t+\Delta t]$ is such that the conditional expectation $E\{y(t+\Delta t) \mid y(t)\}$ of $y(t+\Delta t)$ for fixed $y(t)$ changes as follows

$$
\frac{E[y(t+\Delta t) \mid y(t)]-y(t)}{\Delta t} \approx y(t)[s \theta-\gamma-\delta-\lambda \mu(y(t))] .
$$

As we can see in Fig. 2 the value $s \theta-\gamma-\delta-\lambda \mu(y)$ is positive in the interval $\left(0, y_{1}\right)$. Therefore, the economy is expected to grow in $\left(0, y_{1}\right)$. If the economy is in interval $\left(y_{1}, y_{2}\right)$, the value $y(t+\Delta t)$ is expected to decrease towards $y_{1}$. The state $y_{1}$ is expected to be a trap, i.e., the economy stagnates around $y_{1}$. If the economy is pushed up to cross threshold $y_{2}$, it enters a path of sustained growth until it reaches the next trap. These conclusions concern only expectations. Nevertheless, it is possible to show that in our economy every random path $y(t)$ converges to a trap with probability 1 . In other words, starting from the same initial conditions, the economy may end up (without assistance to growth) at different traps and stagnate within these traps thereafter. This simple model illustrates that the assistance to growth is very important in such intervals as $\left(y_{1}, y_{2}\right),\left(y_{3}, y_{4}\right)$.

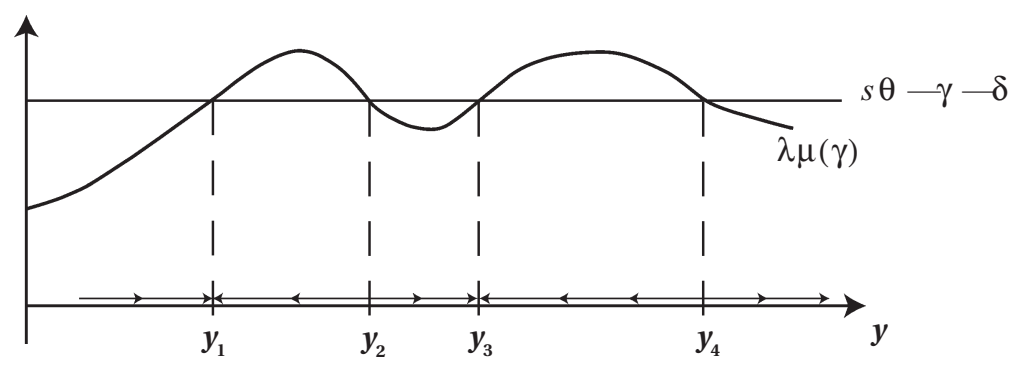

Figure 2. Traps and thresholds. 
If $s, \theta, \gamma, \delta$ depend on $y$, the constant $s \theta-\gamma-\delta$ in Fig. 2 changes to an oscillating curve, but the character of the path dependencies remains the same. The assistance to growth has to change these curves, e.g., to increase the value $s \theta-\gamma-\delta$ and/or decrease $\lambda \mu(y)$ in intervals $\left(y_{1}, y_{2}\right),\left(y_{3}, y_{4}\right)$. Of course, we can not derive a similar to Fig. 2 curve in general cases. Instead, we have to rely only on an optimization model designed with the main purpose to remove traps and thresholds in an optimal way. Consider this with some detail.

\section{Stochastic optimization model}

A simple growth stabilization model of section 3 illustrates the coexistence of adaptive and anticipative decisions. This is a general fact. Consider the following important growth stabilization problem where a part of the growth is used to protect a given target value of the growth rate. According to the model (6) the growth rate $g=s \theta-\gamma-\delta$ may be interrupted by a shock $\omega$. In order to protect $g$ it is possible to mobilize internal resources by reducing consumption, increasing inflation, by asking for external aid and borrowing. Let us denote this type of decisions by $z(\omega)$, where $\omega$ indicates that they are made after observation of the shock $\omega$. Besides, it is possible to reduce or spread losses through various ex-ante measures. Let us denote this type of decisions by $x$. They are chosen before the observation of $\omega$ and, therefore, they do not depend on $\omega$. In general, $x$ and $z(\omega)$ are vectors. For example, the first component of $x$ may correspond to a measure for the reinforcement of buildings, second - to an insurance contract, another pair of components - to a weather related bond characterized by two decision variables specifying its "trigger" and the "cap". The fundamental challenge with the presence of ex-ante strategies in contrast to ex-post strategies is that they must be evaluated not only against the observed $\omega$ but against all possible $\omega$. This requires specific computational approaches. Consider a discrete time version of (6). Assume that $G(t)=\ln y(t)$ and a time horizon [0,T] consists of $N$ subintervals of length $\Delta t=T / N, t_{s}=t_{s-1}+\Delta t, s=1,2, \ldots N, t_{0}=0$. We also assume that a shock $\omega$ at time $t=t_{s}$ comprises all events in $\left[t_{s}-\Delta, t_{s}\right]$. In the same manner losses $v_{t}, t=t_{s}, s=1,2, \ldots, N$ from $\omega_{t}$ comprise aggregate losses from all events in $\left[t_{s}-\Delta, t_{s}\right]$. For the simplicity of notations we assume that $v_{t}$ may be equal 0 and $\Delta t=1$, i.e., we simply consider time moments $t=0,1, \ldots, N$. Variables $G(t), v_{t}$ in general depend on the history of shocks $\omega^{t}=\left(\omega_{1}, \ldots, \omega_{t}\right)$. In the following we use the notations $G(t, \omega), v_{t}(\omega)$ to indicate this dependence, where $\omega=\left(\omega_{1}, \ldots, \omega_{N}\right)$. A vector $x$ of ex-ante decisions 
chosen at $t=0$ transforms $v_{t}(\omega)$ into $v_{t}(x, \omega)$, whereas $G(t)$ depends on both ex-ante and ex-post decisions $G\left(t, x, z^{t}(\omega), \omega\right), z^{t}(\omega)=\left(z_{1}(\omega), \ldots, z_{t}(\omega)\right)$. By using these notations we can write the discrete time version of (6) in the form

$$
G\left(t, x, z^{t}, \omega\right)=G\left(t-1, x, z^{t-1}, \omega\right)+g t-v_{t}(x, \omega)+z_{t}-\left(1+\beta_{t-1}\right) z_{t-1},
$$

where $g=s \theta-\gamma-\delta, t=1, \ldots, N, x \in X \in R^{n}$ and $X$ is a compact set of feasible ex-ante decisions, $z_{t}$ is the amount of borrowing (credits) in $[t-1, t], z^{t}=$ $\left(z_{0}, z_{1}, \ldots, z_{t}\right)$. In this model we assume that credits at $t$ are paid at $t+1$ with the interest rate $\beta_{t}$, which may require borrowing at $t+1$, and so on.

The problem is to find a combination $(x, z(\omega))$ of ex-ante and ex-post decisions maximizing the expected accumulated growth rate in $[0, T]$, i.e., the expectation function

$$
E\left[G\left(T, x, z^{T}(\omega), \omega\right)-\left(1+\beta_{T}\right) z_{T}(\omega)\right],
$$

ensuring a given target growth rate $q$ :

$$
G\left(t, x, z^{t}, \omega\right) \geq q t, t=1,2, \ldots, T, \text { for all } \omega .
$$

In general, $q$ may also be a decision variable, but here we assume that $q$ is fixed. We can subtract $q$ from $g$ in (11) and transform (13) into requirements

$$
G\left(t, x, z^{t}, \omega\right) \geq 0, t=1,2, \ldots, T \text {, for all } \omega .
$$

Therefore, in the following we consider (14) instead of (13), assuming $g=s \theta-\gamma-\delta-q$. In this model a target value $q$ of the growth rate is achieved by sacrificing a portion of the economic growth for defensive ex-ante and expost measures. Let us note that $G\left(t, x, z^{t}(\omega), \omega\right)$ is a linear in $z^{t}(\omega)$ function and it may also be a linear function in $x$. The important feature of the model (11), (12), (14) is that it incorporates both ex-ante $x$ and ex-post $z(\omega)$ decisions, which generates a strong risk aversion with respect to $x$. Indeed, $G\left(t, x, z^{t}(\omega), \omega\right)$ can be written in the form

$$
G\left(t, x, z^{t}(\omega), \omega\right)=\bar{G}_{t}(x, \omega)-\sum_{s=1}^{t-1} \beta_{s} z_{s}+z_{t}
$$


where $\bar{G}_{t}(t, \omega)=\ln y_{0}+g t-\sum_{s=1}^{t-1} v_{s}(x, \omega)$. From this it follows that $z_{t}$ satisfies equation

$$
z_{t}=\max \left\{0,-\bar{G}_{t}+\sum_{s=1}^{t-1} \beta_{s} z_{s}\right\} .
$$

Therefore, the problem (11), (12), (14) can be rewritten as the maximization of the expectation function

$$
F(x)=E f(x, \omega),
$$

which depends only on ex-ante decisions $x \in X$, where

$$
\begin{aligned}
f(x, \mathrm{w})= & \bar{G}_{T}+\beta_{1} \min \left\{0, \bar{G}_{1}\right\}+\beta_{2} \min \left\{0, \bar{G}_{2}+\beta_{1} \min \left\{0, \bar{G}_{1}\right\}\right\}+ \\
& \beta_{3} \min \left\{0, \bar{G}_{3}+\beta_{1} \min \left\{0, \bar{G}_{1}\right\}+\beta_{2} \min \left\{0, \bar{G}_{2}+\beta_{1} \min \left\{0, \bar{G}_{1}\right\}\right\}\right\}+\ldots
\end{aligned}
$$

In other words, the problem (11), (12), (14) is equivalent to the maximization of the nonlinear in $x$ expectation function (16) and the calculation of expost decisions according to the recursive equation (15). If the probability distribution of $v_{t}$ for some $t=1, \ldots, N$ has a continuous density function, then the expectation function (16) is a strictly concave function since $f(x, \omega)$ is formed by using the operation min of linear in $x$ functions. The described above implicit nonlinear character of the problem (11), (12), (14) with respect to is due to the nature of ex-post decision $z(\omega)$. It is made on the basis of $\omega$ and given $x$, i.e., $z(\omega)$ depends implicitly on $x$. This is a general feature of the two-stage stochastic optimization problems (Ermoliev \&Wets, 1988). The model (11), (12), (14) is a dynamic two-stage stochastic optimization problem, which can be solved by specific stochastic optimization procedures.

\section{Concluding remarks}

Any rational strategy for managing the increasing vulnerability of interacting socio-economic and natural systems requires understanding of involved risks and losses. Now existing catastrophe models (Walker, 1997) primarily deal with the estimation of direct losses from natural catastrophes. Indirect losses may be much higher. They include costs of business interruptions, jumps of prices, booms in some sectors and depression in others. A destruction of capital stock results in losses of outputs and, hence, affects wages, profits, savings and investments. This paper shows that shocks may 
have even deeper indirect consequences - they implicitly modify the economy and may cause the stagnation and shrinking even in the case of sustained on average exponential growth.

In our conceptual model the increasing endogeneously generated vulnerability is characterized by a probability distribution in the interval $[0, y]$, which depends on the current level of per capita output $y$. For example, a dam may trigger new economic developments in flood-prone areas but its failure, say, due to an earthquake, may lead even to more dramatic catastrophic losses. Of course, any realistic model of the economic growth under shocks must be based on geographically explicit catastrophe models with specific (for a given economy) patterns of catastrophes and the vulnerability (see discussion in Ermoliev et al., 2000).

Often the costs of catastrophes are borne by the central government, what may "unexpectedly" divert resources from other planned projects or may require "unplanned" borrowing. Anticipative ex-ante hazard preparedness and loss reduction measures may significantly reduce "unexpected" costs of post-shock responses. Any short term adaptive incremental analysis of economic growth underestimates the impacts of rare catastrophic shocks. To purchase catastrophe insurance or bond would be a good solution if the catastrophe occurs tomorrow. But it may occur in 5, 20, or 100 years, i.e., within a time interval sufficient for implementing certain loss reduction measures. The outlined in section 5 model incorporates both ex-ante and ex-post growth efforts and provides a unified decision-making framework to assess benefits of their synergy. From the formal point of view the analysis of the long-term growth efforts is similar to the design of insurance portfolios in the presence of catastrophic risks (Ermoliev et al., 2000). Stochastic models (Davis, 1984), (Daykin, 1994) of a non-diffusion type provide appropriate modeling framework. In both cases the main problem concerns the protection of growth of certain accumulation processes such as risk reserves of insurers or outputs of the economy. The presence of only ex-post options significantly simplifies the analysis, since such measures have to be optimal with respect to a particular observed shock. An ex-ante solution has to be optimal (robust), in a sense, against all possible shocks. The presence of exante options makes impossible to use the so-called recursive equations of the conventional control theory (Sargent, 1987). Major challenges are connected with the lack of historical data on rare events and analytically intractable structures of realistic accounting models for growth processes (MacKellar \& Ermolieva, 1999). The role of catastrophe modeling (Walker, 1997) becomes essential. The most promising approach for managing the 
sustained growth seems to be the use of sophisticated computational techniques of the stochastic optimization. This is becoming increasingly important in financial planning (Dupacova, 1995), (Zenios, 1993), (Ziemba, 1998).

\section{References}

Arrow J. (1996): “The Theory of Risk-Bearing: Small and Great Risks”. Journal of Risk and Uncertainty 12, 103-111.

Climate Change and Increase in Loss Trend Persistence (1999). Press Release, Munich Re., Munich.

Davis M.H.A. (1984): "Piecewise-deterministic Markov Processes: A General Class of Non-diffusion Stochastic Models”. J.R. Statist. Soc. B 46. pp. 353-388.

Daykin C., Pentikainen T. \& Pesonen M. (1994): Practical Risk Theory for Actuaries, Monographs on Statistics and Applied Probability. Vol. 53. Chapman \& Hall Ltd.

Dupacova J. \& Bertocchi M. (1995): "Management of Bond Portfolios via Stochastic Programming - Postoptimality and Sensitivity Analysis”. In: Dole J. \& Fidler J. et al. (eds.): System Modeling and Optimization. Proc. of the 17-th IFIP TC7 Conference, Prague. Chapmen \& Hall. pp. 574-582.

Easterly W. (1994): "Economic Stagnation, Fixed Factors, and Policy Thresholds". Journal of Monetary Economics 33. pp. 525-557.

Ermoliev Y.M., Ermolieva T.Y., MacDonald G. \& Norkin V.I. (2000): “Insurability of Catastrophic Risks: the Stochastic Optimization Model”. Optimization, Vol. 47 2000. pp. 251-265.

Ermolieva T.Y., Ermoliev Y.M. \& Norkin V.I. (1997): “Spatial Stochastic Model for Optimization Capacity of Insurance Networks under Dependent Catastrophic Risks: Numerical Experiments”. Interim Report IR-97-028, Int. Institute of Applied System Analysis, Laxenburg, Austria. Download from website <www.iiasa.ac.at $>$.

Ermoliev Y., Ermolieva T., MacDonald G., Norkin V. \& Amendola A. (2000): “A system approach to management of catastrophic risks". European Journal of Operation Research 122. pp. 452-460.

Ermoliev Y. \& Wets R. (eds.) (1988): "Numerical Techniques of Stochastic Optimization”. Computational Mathematics. Springer-Verlag.

Khan M.S., Montiel P. \& Haque N.V. (1990): "Adjustments with Growth: Relating the Analitical Approaches of the IMF and the World Bank". Journal of Development Economics 32, 155-179.

MacKellar L. \& Ermolieva T. (1999): “The IIASA Multiregional Economic-Demographic Model: Algebraic Structure”. Interim Report IR-99-007, Int. Institute of Applied Systems Analysis, Laxenburg, Austria. Download from website <www. iiasa.ac.at>. 
Ray D. (1998): Development Economics. Princeton, NJ: Princeton University Press. Sargent T. J. (1987): Dynamic Macroeconomic Theory. Cambridge, MA: Harvard University Press.

Solow R. (1997): Growth Theory, An exposition. Oxford: Clarendon Press.

Walker G. (1997): “Current Developments in Catastrophe Modelling”. In: Britton N.R. \& Oliver J. (eds.): Financial Risks Management for Natural Catastrophes. Brisbane, Australia: Griffith University. pp. 17-35.

Zenios S.A. (ed.) (1993): Financial Optimization. Cambridge, MA: Cambridge University Press.

Ziemba W.T. \& Mulvy J. (eds.) (1998): World Wide Asset and Liability Modeling. Cambridge, MA: Cambridge University Press. 



\section{Additional copies}

Further copies of this IIASA Reprint are available online at www.iiasa.ac.at/Publications

Hard copies are also available for a small handling charge. Orders must include the publication number and should be sent to the Publications Department, International Institute for Applied Systems Analysis, A-2361 Laxenburg, Austria.

Telephone: +432236807

Telefax: +432236 71313

E-mail: publications@iiasa.ac.at 
International Institute for Applied Systems Analysis

Schlossplatz 1, A-2361 Laxenburg, Austria

Tel: +432236807 Fax: +43223671313

I I AS A www.iiasa.ac.at 